\title{
Optimization of Ant based Cluster Head Election Algorithm in Wireless Sensor Networks
}

\author{
Siddharth Kumar \\ M.Tech Student, \\ Dept of Computer Science and Technology, \\ Central University of Punjab, \\ Punjab, India
}

\begin{abstract}
Wireless Sensor Networks consist of inexpensive nodes that are positioned over an area to collect the useful information and send the valuable data to the base station for further processing. In wireless sensor networks, nodes have limited power and shorter lifetime. So, it is crucial to aggregate the data in Energy Efficient manner and optimize the lifetime of the network. To solve the issue, Ant Colony Optimization, a Swarm Intelligence based Routing Technique is used. The proposed system is a Routing Approach based on ACO Algorithm along with the Clustering approach in which Cluster Head is elected based on maximum value of energy and degree to maximize Energy Efficiency of the network and to increase the lifetime of the network. Performance obtained from the simulation results shows that the proposed approach provides an Optimized solution in terms of Enhanced Network Lifetime and Efficient Energy Utilization.
\end{abstract}

\section{General Terms}

Optimization, Cluster Head, Energy, Degree, Efficiency, Packet Delivery Ratio

\section{Keywords}

Single hop packet progress, Next hop, Optimized ACO, Existing ACO

\section{INTRODUCTION}

A Wireless sensor network (WSN) is a collection of a vast number of sensor nodes, which are deployed in an area that requires supervision. Some of the applications of the WSN are health, military, and security. The sensor nodes communicates with each other through the wireless links, and it uses radio frequency channels for communication. Sensors nodes are equipped with a processor, small storage memory, and a communication channel.

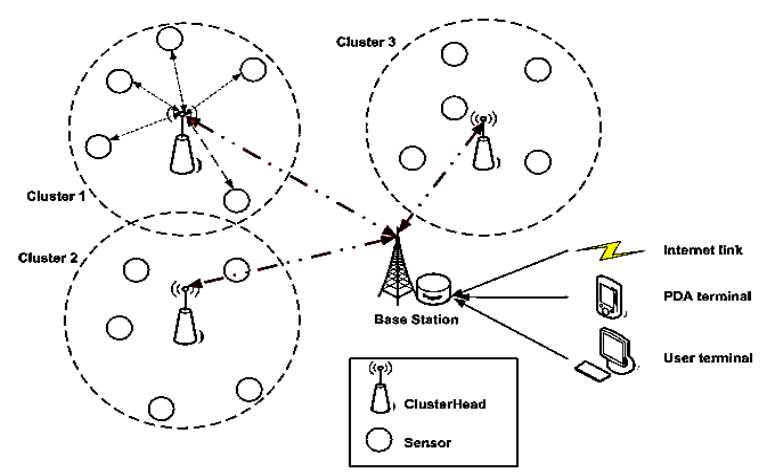

Figure 1: Wireless Sensor Network
The wireless sensor networks have been studied thoroughly in the recent years. Sensors nodes are spread in a specified area. It collects the data from the environment and sends it to the base station for further processing [1]. Base station is a data sink, and it has more processing power, storage space, and capability of communicating with the sensors.

The sensor nodes have limited storage and power, limited computational ability. It comprises of four parts namely: transceiver, processor, battery and most importantly a sensor also known as motes/ nodes. Routing is defined as the way of finding the shortest path between two nodes. For many years ants have successfully applied routing to find the shortest paths between the food sources and their nest by means of a pheromone trail laid by the other Ants this is known Ant Colony Optimization (ACO) routing[2].

An ant in a colony uses a volatile chemical substance called pheromone to converge to the shortest path among multiple paths that connect their nest to the food source. While moving, ants release pheromone on the ground, and they follow the path of maximum pheromone concentration. This mechanism provides an optimum way to mark paths that guide other ants, and generate right paths from the overall behavior of the ant colony. The principle objective of this technique is to provide maximum network lifetime while finding the shortest paths between source and the base station using ACO technique, which is swarm intelligence based optimization technique.
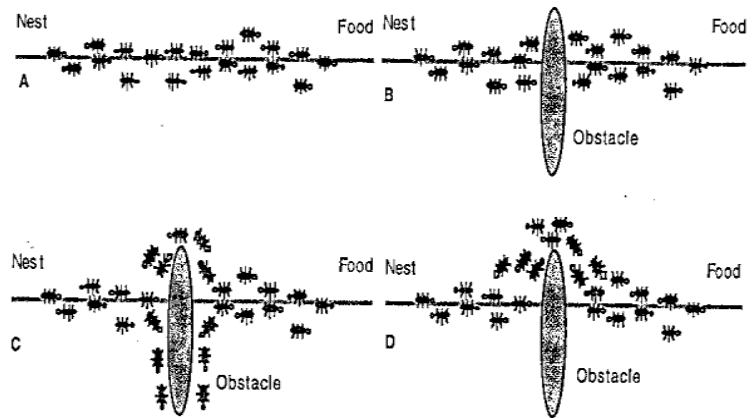

Figure 2: Simple Ant Colony Optimization Technique (A) Ants uses a direct path between nest and food. (B) An obstacle hinders the path. (C) Pheromone is deposited quickly on the shortest path. (D) After sensing the high pheromone, all the ants choose the shortest path

It includes a collection of asynchronous agents or ants that produces partial solutions while moving through different problem states. While moving they follow a decision policy that is based on two parameters namely trail information and attractiveness. Each ant while moving incrementally produces a solution. When the final solution is generated, the path 
information of the components is modified by evaluating the solution that will help in directing the further mechanism of the future ants.

\section{RELATED WORKS}

\subsection{Pheromone Based Energy Aware Directed Diffusion (PEADD)}

PEADD [3] is based on Ant Colony Optimization technique. This protocol aims at maximizing the lifetime of the sensor nodes by deploying nodes having higher energy for the information gathering process. In this algorithm, ant agent increases the pheromone value on a path corresponding to the remaining energy levels of nodes. Paths with higher residual energy is elevated. The value of pheromone decays with the transmission of data because the pheromone value is linked to the remaining energy. The pheromone value is revised based on the amount of transmittied data.

The simulation results show that for an increased number of dead nodes, the network lifetime attained by this protocol is much higher as compared to the Directed Diffusion.

\subsection{Probabilistic, Zonal and Swarm- Inspired System for Wildfire Detection (PZSWiD)}

This protocol follows a data-centric approach; the system follows a swarm inspired routing and aggregation algorithm. This algorithm uses a probabilistic model for representing information in a data-centric sensor network.

In PZSWiD, the sensor nodes perform two functions firstly they reply to different queries generated by a sink node, and secondly they transport detected events, e.g., an outbreak of fire to the sink. The algorithm was simulated in NS-2 with a variation of different zone radii, while analyzing its average delay and the average energy dissipated. This algorithm has not been compared with any existing protocol for performance measurements [4].

\subsection{Adaptive Clustering For Energy Efficient WSN Based On ACO (ACO-C)}

[5] Proposed ACO-C which is based on ACO it uses adaptive clustering for energy efficient wireless sensor networks. This algorithm uses a new energy-aware clustering approach by using appropriate cost function implemented at the base station. It minimizes the cost of long distance transmission and distributes the data among all sensor nodes evenly using aggregation. The routing problem was adapted as a clustering problem in which the objective is to select $\mathrm{K}$ out of $\mathrm{N}$ nodes as cluster heads, which was achieved through agent consideration called software ants. The simulation of algorithm was carried in Matlab platform and compared with LEACH. Protocols like LEACH-C and PSO-C performed better in terms of data delivery and network lifetime.

\subsection{Ant Colony Clustering Algorithm (ACALEACH)}

[6] Suggested an Ant Colony Clustering Algorithm. Which is an improved version of LEACH. This algorithm considers the node residual energy and the distance between the cluster heads for selection of cluster heads. It uses the ant colony algorithm into the inter-cluster routing technique which reduces the energy consumption of cluster heads and thus optimises the lifetime of the sensor networks.

This algorithm is compared with LEACH in Matlab environment in which it outperforms in terms of average energy consumptions and survival nodes at the end of the simulation. The protocol does not consider throughput and delay in its routing process thus it is weak in energy efficiency due to extra overheads.

\subsection{Energy Efficient Ant Based Routing (EEABR)}

EEABR [7] is based on Ant Colony Optimization (ACO) technique. In this protocol, each node in the network launches a forward ant at regular intervals with the aim of inventing a route to the base station. In this protocol, each ant only carries the address of the last visited nodes. When a node receives a forward ant, it searches it in the table for any possible loop. If there is no loop, then the node saves into its table the information of the ant and restarts the timer and forwards it to next hop. When the forward ant reaches the destination, it is converted to backward ant with the mission to renew the pheromone trail of the path traversed by the forward ant

When compared to basic ant-based routing (BABR) and Improved Ant-Based Routing (IABR), it performs superior in terms of average energy of nodes, the energy of node with minimum energy and energy efficiency. The disadvantages are that its shortfall in the quality of service and increases the excessive delay in packet delivery.

\subsection{Energy Efficient ACO Based QoS Routing (EAQR)}

Proposed energy efficient ACO based QoS routing (EAQR), it is improved version of ant colony optimization algorithm. This protocol provides preference to QoS and balancing of energy consumption over the entire network. It introduces minimum path energy, path hop count, and by means of advancing pheromone trail model of the ant colony system. It provides two heuristic ways based on the length and the comfort of the path to meet the different performance requirements of real-time and common traffics. It also provides the service of differentiation between real time and best effort traffic by introducing the new dual pheromone heuristic model in the ant colony system [8].

\subsection{An Adaptive QoS and Energy Aware Routing Algorithm (AEARA)}

[9] Proposed an Adaptive QoS and Energy-aware Routing Algorithm (AEARA) which is an improved version of Antbased Routing Algorithm. This protocol mainly focuses on QoS with balancing the energy utilization of each node to prolong the lifetime of the network. This algorithm consists of two parts, the route discovery and route maintenance. A routing table is maintained and timely response to topology changes. Due to limited availability of bandwidth, this algorithm is more crucial for real time high bandwidth traffic requirement such as in multimedia transmissions.

\subsection{Ant Based Cluster Head Election Algorithm in Wireless Sensor Network to Avoid redundancy in WSN}

In this paper, a novel routing approach based on ACO Algorithm in Wireless Sensor Networks is applied which is used to route the data packets in sensor networks to maximize energy efficiency and to increase the network lifetime. It also reduces the efforts used in sending the redundant data sent by the sensors that are close to each other in the sensor network [10]. The performance of proposed algorithm is compared with the LEACH protocol, and the simulation results show that the proposed approach provides optimized solutions in 
terms of efficient energy utilization and enhanced network lifetime.

\section{EXISTING SYSTEM}

The existing system [10] is the sensor network sensor nodes are grouped into clusters controlled by a single base station. Each cluster consists of a cluster head node that manages sensors in the cluster. The existing ant-based approach, there are two phases set-up phase and steady state phase as in LEACH protocol. The cluster head is chosen randomly in Existing ACO protocol for data transmission. ACO technique is applied in sending the packet data from these sensor nodes to the cluster head. This routing algorithm uses the probabilistic approach for constructing the solution, i.e. selecting the suitable path for transmission. This probabilistic selection uses pheromone information and the heuristic information, which is updated continuously. Data is transmitted from the sensor to the cluster head that is selected randomly through the optimal path.

$$
\mathrm{P}_{\mathrm{ij}}=\frac{\mathrm{SPP} * \mathrm{E}}{\sum(\mathrm{SPP} * \mathrm{E})}
$$

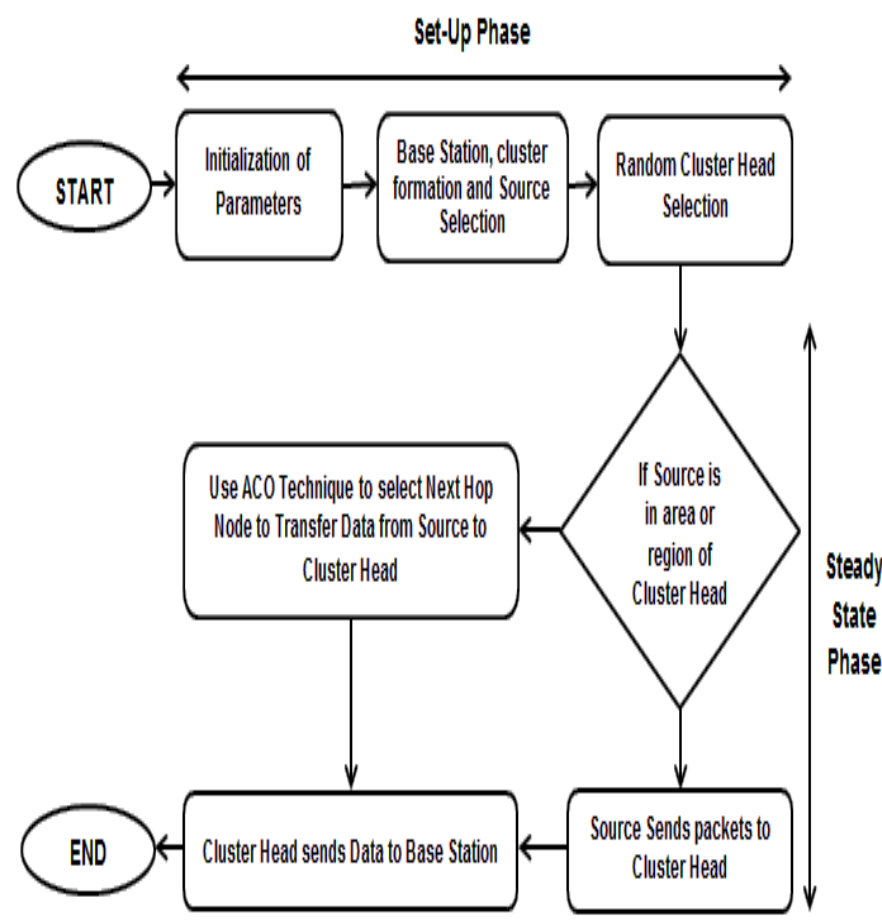

Figure 3: Flow Chat of Existing ACO Protocol

Single Hop Packet Progress (SPP) depends upon the difference between the distance of source node to base station node and distance of an adjacent node to base station node. Energy (E) for each node is predefined. Probability is calculated for each node in the clusters and the node having highest probability will be selected as next hop for data transmission from source node to the cluster head which was elected randomly.

\subsection{Disadvantages}

It is not energy efficient as cluster head is chosen randomly among the live sensors, which leaves the scope of efficient selection of cluster head that will optimize the efficiency of the overall network.

\section{PROPOSED SYSTEM}

The existing work [10] is not efficient as cluster head is chosen randomly among the live sensors, which leaves the scope of efficient selection of cluster head that will optimize the efficiency of the overall network. The proposed work makes use of maximum value of product of degree and energy for cluster head election.

In ACO technique, the ant agents are placed on the source node that iteratively produces the solution by using probabilistic approach and the pheromone value of optimum path from source to sink. For all the nodes that lie outside the radius of Cluster Head, ACO is applied in sending the packet data from these sensor nodes to the cluster head. This routing algorithm follows the probabilistic approach for constructing the solution, i.e. selecting the suitable path for transmission. Hence, the drain rate of the nodes will be linear, and packet delivery rate will be increased. Hence, network lifetime will be prolonged in higher level compared to other algorithms.

\subsection{ADVANTAGES}

- Proposed approach provides improved packet delivery rate network lifetime

- It modifies existing ACO protocol by selecting cluster head efficiently among live sensors.

\section{ENHANCEMENT}

In Optimized system the sensor nodes are grouped into clusters controlled by a single base station. Each cluster consists of a cluster head node that manages sensors in the cluster. In Optimized ant-based approach, there are two phases set-up phase and steady state phase as in LEACH protocol. The cluster head is chosen based on the maximum value of energy and degree for data transmission.

$$
\text { Cluster Head }=\text { Max }\left(\text { Degree }^{*} \text { Energy }\right)_{\mathrm{i}=1 \mathrm{toN}}
$$

The selection of cluster head based on energy and degree improves the network lifetime and reduces the communication cost. ACO technique is applied in sending the packet data from these sensor nodes to the cluster head. This routing algorithm uses the probabilistic approach for constructing the solution, i.e. selecting the suitable path for transmission. This probabilistic selection uses pheromone information and the heuristic information, which is updated continuously.

$$
\frac{\mathrm{P}_{\mathrm{ij}}=\mathrm{SPP} * \mathrm{E}}{\sum(\mathrm{SPP} * \mathrm{E})}
$$

Single Hop Packet Progress (SPP) depends upon the difference between the distance of source node to base station node and distance of an adjacent node to base station node. Energy (E) is predefined for each node. Probability is calculated for each node in the clusters and the node having highest probability will be selected as next hop for data transmission from source node to the cluster head which was elected randomly. 


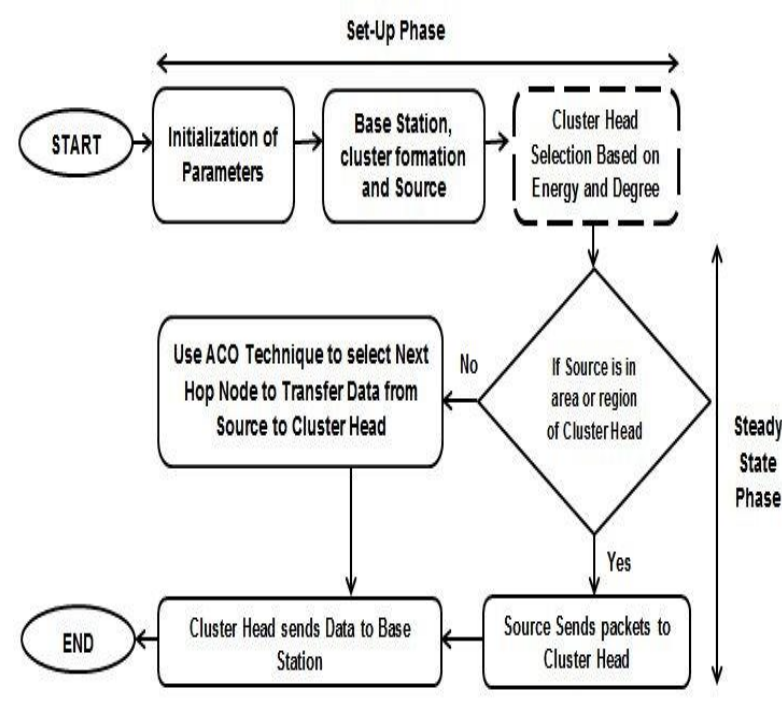

Figure 4: Flow Chart of Optimized ACO Protocol

\section{SIMULATION PARAMETERS AND RESULTS}

In simulation, we have compared the performance of Existing [10] and Optimized ACO protocol. These sensor nodes are distributed in $600 * 600$ square. These two protocols are compared considering:

1) The total number of nodes alive within the system at different rounds of iteration.

2) The total energy of the system at different rounds of iterations.

3) The total number of packets transmitted to the base station at different rounds of iterations.

Table 1: Parameter values used for simulation

\begin{tabular}{|l|l|}
\hline Simulator & Network Simulator 2 \\
\hline Topology & Random \\
\hline Interface Type & Phy/ WirelessPhy \\
\hline Mac Type & 802.11 \\
\hline Queue Type & Drop tail/Priority Queue \\
\hline Queue Length & 500 Packets \\
\hline Antenna Type & Omni Antenna \\
\hline Propagation Type & Two ray Ground \\
\hline Routing Protocol & AODV \\
\hline Application Agent & CBR \\
\hline Number of Nodes & $20,30,40,50,60$ \\
\hline Simulation Time & 30 ms \\
\hline Packet Size & 512 Bytes \\
\hline Area Size & $600 * 600$ \\
\hline
\end{tabular}

Simulation has shown that the proposed protocol has higher stability period and better energy utilization as compared to
Existing ACO protocol [10]. From the simulation following comparative results are obtained:

\subsection{Comparison of Number of Nodes Alive}

Simulation results show that the Optimized ACO protocol has more number of alive nodes at different rounds of iterations as compared to Existing ACO protocol. As the cluster, head is chosen efficiently in Optimized ACO protocol that makes the proper use of energy for routing data, and thus less number of nodes die in the system as compared to the Existing ACO Protocol.

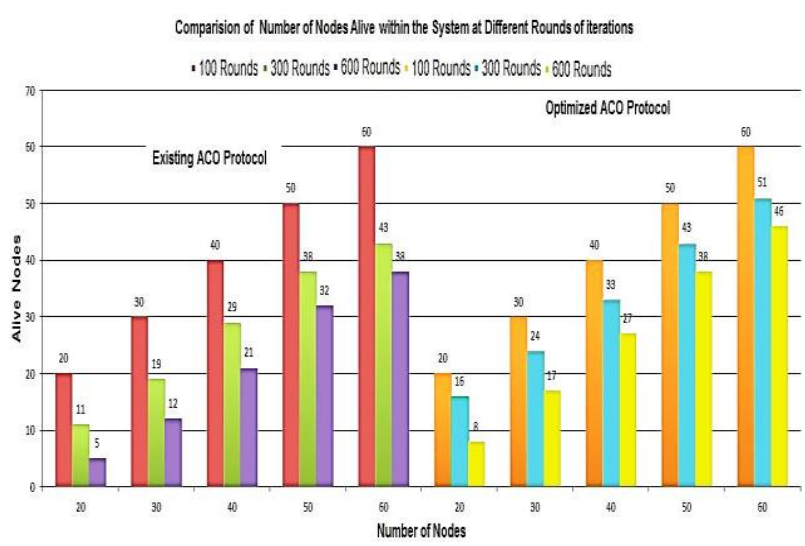

Figure 5: Comparison graph for Number of Nodes alive at different rounds of iterations for Existing and Optimized ACO Protocol

\subsection{Average Residual Energy Comparison}

Simulation result shows that as the number of nodes increases the average residual energy also increases for both Existing and Optimized ACO protocol for the first round of iteration. As shown in figure 6 the drain rate of network energy is decreased in Optimized ACO protocol as compared to Existing ACO Protocol because cluster head is elected based on energy and degree in Optimized ACO Protocol, so the drain rate of the networks energy becomes linear which is not in case of Existing ACO protocol. Thus, it is analyzed that the Optimized ACO protocol is more energy efficient than Existing ACO protocol.

\section{Comparision of Total Energy of the system at Ditierent Rounds of terations

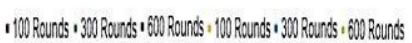

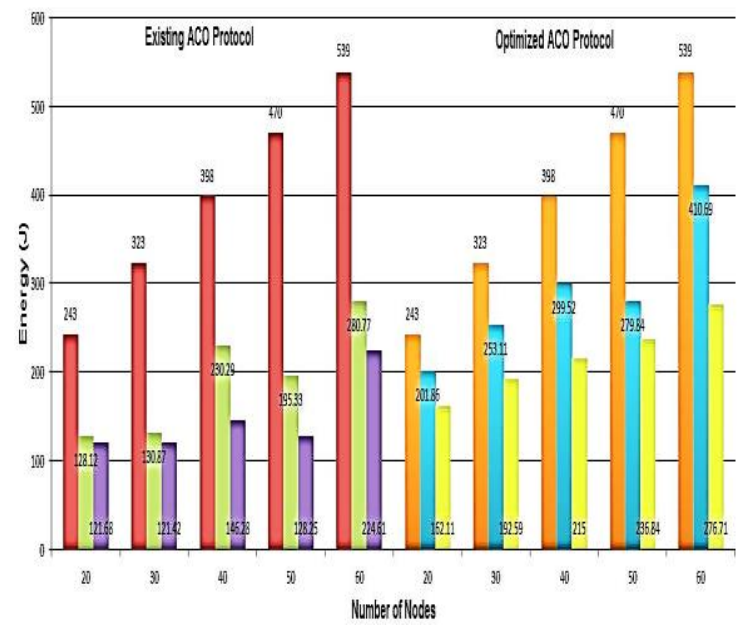

Figure 6: Comparison graph for Residual Energy at different Rounds of Iteration for Existing and Optimized ACO protocol 


\subsection{Number of Packets Transmitted Comparison}

The simulation results show that the Optimized ACO protocol transmits more number of packets in the network as compared to Existing ACO Protocol. In Optimized ACO protocol, cluster head is chosen efficiently based on energy and degree, so packets are routed efficiently for more number of rounds than the Existing ACO protocol.

\section{CONCLUSION}

In proposed Optimized ACO protocol, the cluster head is chosen based on the maximum value of degree and energy. The performance analysis of the Existing and Optimized ACO protocol was carried out, and results show that Optimized ACO protocol has 12.05 percent more number of alive nodes in the network as compared to Existing ACO protocol. The average residual energy of the network is increased by 22.30 percent than the Existing ACO protocol and 32.75 percent more number of packets are transmitted in Optimized ACO protocol.

In a nutshell, it is concluded that the proposed approach provides optimized solutions in terms of average residual energy consumption, number of alive nodes and number of packets transmitted.

\section{FUTURE WORK}

As a future work, Optimized Ant based Cluster Head Election Algorithm can be implemented in the real time scenario. This technique is Energy-efficient in routing of Data packets transmission from source to the base station. To make it more effective, Multi-Hop Routing can be used for Data transmission using ACO technique.

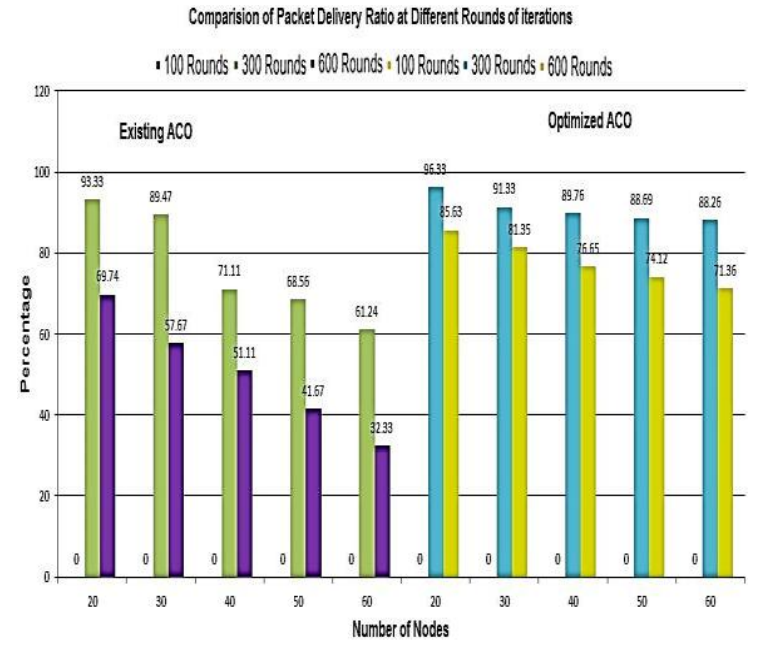

Figure 7: Comparison graph for total number of packets transmitted at different Rounds of Iterations for Existing and Optimized ACO Protocol

\section{REFERENCES}

[1] Yick, J., Mukherjee, B., \& Ghosal, D. (2008). Wireless sensor network survey. Elsevier, 52(2008), 2292-2330.

[2] Zungeru, A. M., Ang, L.-M., \& Phoo, K. (2012). Classical and swarm intelligence based routing protocols for wireless sensor networks: A survey and comparison. Elsevier, 35(2012), 1508-1536.

[3] Zhu, X. (2007). Pheromone based energy aware directed diffusion algorithm for wireless sensor network. Proceedings of the international conference on intelligent computing (pp. 283-291). ICIC.

[4] Ramachandran, C., Misra, S., \& Obaidat, M. (2008). Probabilistic zonal approach for swarm inspired wildfire detection using sensor networks. International Journal of Communication Systems, 1047-1073.

[5] Ziyadi, M., Yasami, K., \& Abolhassani, B. (2009). Adaptive clustering for energy efficient wireless sensor networks based on ant colony optimization. Proceedings of the seventh annual communication networks and services research conference, (pp. 330-334) .

[6] Wang, G., Wang, Y., \& Tao, X. (2009). An ant colony clustering routing algorithm for wireless sensor networks. Proceedings of third international conference on genetic and evolutionary computing (pp. 670-673). ICGEC.

[7] Camilo, T., Carreto, C., Silva, J., \& Boavida, F. (2006) An energy efficient ant-based routing algorithm for wireless sensor networks. IEEE, 49-59.

[8] Jietai, W., Jiadong, X., \& Mantian, X. (2009). EAQR: an energy-efficient ACO based QoS routing algorithm in wireless sensor networks. Chinese Journal of Electronics, 113-116.

[9] Peng, S., Yang, S., Gregori, S., \& Tian, F. (2008). adaptive QoS and energy-aware routing algorithm for wireless sensor networks. IEEE2008, 578-583.

[10] Sharma, T., Kumar, B., Berry, K., Dhawan, A., Rathore, R. S., \& Gupta, V. (2014). Ant Based Cluster Head Election Algorithm in Wireless Sensor Network to avoid Redundancy. IEEE, 1(2014), 83-88. 